\title{
Solar Energy, Public Utilities, and Economic Efficiency*
}

\author{
WILLIAM HAMLEN \\ State University of New York at Buffalo \\ Buffalo, New York \\ JOHN TSCHIRHART \\ University of Wyoming \\ Laramie, Wyoming
}

\section{Introduction}

There is general agreement that solar radiation will one day be an important source of energy. There is considerable disagreement as to when this energy source will be supplying a substantial percent of the world's needs. The two principle areas where solar energy could become instrumental are in heating and in the production of electricity. In recent years significant advances have been made in heating, to the point where numerous firms are marketing solar heating systems. On the other hand, solar electricity is still years away from widespread use. The first solar-electric plant should be ready to generate around 1985.'

Common to both technologies are the same basic components: a solar collector; a storage facility; and a conventional backup system. These components form the capital stock while solar radiation is the fuel, along with a conventional backup fuel. As a fuel, however, solar radiation is unusual because of its random availability, causing ramifications for designing economically efficient systems, and for interfacing these systems with existing public utilities. The objective in this paper is to present and interpret a model that captures the basic elements of a solar energy system, including the random elements on both the demand and supply sides of the market. A chance-constrained cost minimization technique is used to determine the economically efficient combination of capital inputs. In addition, the problem of interfacing this system with existing technologies is examined.

Because of the similarities between the heating and electricity technologies, the model presented is couched in terms of heating only. With minor changes, the model could be

\footnotetext{
* We are indebted to William Schulze and an anonymous referee for very valuable suggestions and comments on an earlier draft. Our thanks also go to Donna Lake for her editorial assistance. This research was partially supported by Los Alomos Scientific Laboratories Grant Number N28-8365H and our thanks go to Fred Roach for his suggestions. Support was also obtained from a Dean's Summer Fellowship School of Management, SUNYAB, and the Research Foundation of SUNY. Any errors are our responsibility.

1. This projection and a description of solar-electric technologies can be found in Caputo [5].
} 
adapted to electricity as well. A number of studies have explored the economic aspects of solar heating systems in an attempt to determine when solar energy will be an economically viable alternative to traditional energy sources. Tybout and Löf [26] make projections of solar heating costs for various U.S. cities by optimizing the heating system design for each location. They then compare these costs to the costs of conventional heating, and they also design a least-cost combination of solar and conventional heating technologies. Ben-David et. al. [4] extended this work by taking into account the OPEC phenomena and the depleting stocks of oil and natural gas reserves, which will have a substantial influence on future energy prices. They also employ a model that derives a least-cost combination of technologies and discuss the competitiveness of solar energy versus conventional energy sources for the entire U.S. In more recent work, Roach, Noll, and Ben-David [19] conclude that solar heating is competitive today with electric heating systems in most areas of the U.S.

The accuracy of the predictions made in these papers and others is crucially dependent on the models used to determine the least-cost combination of heating technologies. Unfortunately, the models used have a number of shortcomings. They do not account for the stochastic elements inherent in the problem. The demand for heat depends on stochastic weather conditions, but the above authors use weather data from a "typical" year. The supply of solar radiation is also a stochastic variable; again the previous studies use solar radiation measurements from a "typical" year. Simulation models are used to determine solar heating cost as a function of the fraction of total demand met by the solar system.

Since the heating systems are designed for the "typical" year, an obvious concern is the performance in an atypical year. During severe weather, more conventional fuel will be needed as a backup, but the models cannot assess the probabilities of such an event nor the likely additional costs because of the extra fuel used. Moreover, the implicit assumption is that in the atypical year, the extra fuel needed to meet heat demands is forthcoming. This places the entire burden of uncertainty on the suppliers of conventional heat, i.e., the public utilities. As a result, the public utilities are not in any way relieved of the responsibility of maintaining large capacities to meet emergency conditions. Thus, if solar heating systems lead to less overall demand for the public utility's service, then utility prices must increase to cover a greater average cost. Without an integrated approach among solar users and the public utility, the latter is likely to be an impediment to future expansion of solar energy. ${ }^{2}$ And without specifically accounting for the random elements inherent in the problem, a proper analysis of the interface problem is not possible.

The model formulated herein offers a very different approach that avoids the aforementioned shortcomings. The objective is still to minimize the cost of heating with an active solar system and a conventional backup; however, the stochastic elements are incorporated by recognizing that any given amount of conventional fuel, along with a solar system, can meet heating demands only with a particular probability. The probability is assured through the use of chance constraints. The structure of the problem also alleviates the public utility from the entire burden of uncertainty. This is accomplished through rate structure design. The treatment of solar radiation as a stochastic variable is shown to be particularly important because of the random fluctuations in radiation over successive days. This randomness has been shown to exhibit geometric Brownian motion.

Section II provides a brief description of the solar heating system and the equation repre-

2. See Feldman and Anderson [9] and Schiflett and Zuckerman [22] for a discussion of this point. 
senting the excess supply of heat. In Section III, the public utility interface issue is discussed. Section IV presents the cost minimization model, and in Section $\mathrm{V}$ the results of the model are interpreted. Section VI is a conclusion. Two Appendices provide more detail on the chance constraints, the optimization problem, and geometric Brownian motion.

\section{The Solar Heating System}

A detailed description of a typical active solar space heating system can be found in Tybout and Löf [26] or Kreider and Kreith [14]. In this paper, a brief description of the main components of the system and their interaction will suffice. The components for an individual building include a collector, a storage tank, and an auxiliary heater. The collector is usually placed on the roof of the building. It consists of one or more flat glass plates separated by insulating zones that together cover a black absorbing plate made of metal, plastic or rubber. The glass covers are designed to allow solar radiation to pass through to the absorbing plate, and then prevent the infrared radiation from escaping back into the atmosphere.

The maximum amount of heat, measured in British thermal units (Btu's), that can be supplied by a collector depends on the solar radiation available, weather conditions, and on a variety of technical attributes. These include the transmittance properties of the covers, the absorption capabilities of the absorbing plate, the tilt of the collector surface, and thermal losses due to convection and radiation. To summarize these attributes for a given collector, an efficiency rating, $\eta$, is used which is defined as the ratio of heat output per square meter of collector surface to the heat input from the incident solar radiation. A typical value of $\eta$ would be in the range of $30 \%$ to $40 \%$. Using the efficiency rating, solar radiation is converted to usable heat by the following conversion factor:

$$
b=[\gamma+[1-\gamma] \chi] \eta \text {. }
$$

Here, $\gamma$ and $1-\gamma$ are the diffused and direct portions of solar radiation respectively. The direct portion is multiplied by the tilt factor, $\chi$, which is the angle between the plane of the collector surface and the horizontal. The optimum tilt factor depends on latitude, the position of the sun relative to the collector during the year, and the local terrain. Tybout and Löf find the optimum tilt factor to be latitude plus $15^{\circ}$, however, the performance of the system does not vary much over wide ranges of $\chi$. Finally, the heat generated by the collector during period $t$ is given by

$$
s_{t} b A
$$

where $s_{t}$ is the incoming solar radiation in Btu's per area and time and $A$ is the area of the collector surface. The time period $t$ will be discussed in detail in Section IV.

The heat accumulated by the collector is not used directly, but is first transferred to storage. The temperature of the collector must exceed storage temperature before any transfer occurs. The heat is removed from the collector's absorbing plate by air or water and then carried to the storage medium which usually consists of rocks or water. Rocks are generally used with hot air systems and have the advantage of being more easily contained than water. Heat is removed from storage when the temperature of the rocks or water exceeds some specified amount (about $30^{\circ} \mathrm{C}$ ) and weather conditions require that the building be warmed. The heat capacity of storage is denoted by $S$.

Since storage must be at a minimum temperature to be useful, and since there are thermal losses from storage depending on its volume and insulation, a very large storage capacity 
is wasteful. Alternatively, a very small capacity means high storage temperatures and again significant thermal losses. Any heat that the collector gathers after the maximum temperature is reached is simply lost to overflow. Overflow loss in period $t$ is denoted by $L_{t}$. Thus storage that is too small will not take full advantage of available solar energy.

The demand for heat during any period depends on the outside temperature and on the size and technical specifications of the building's construction. Enough heat must be provided to account for thermal losses through the walls, windows, and roof, and to warm the ventilating air and the air entering by infiltration. A heat-transfer coefficient, $h$, can be determined for the building that will capture these technical specifications. Multiplying the coefficient by the degree day unit for period $t, k_{t}$, yields

$$
k_{t} h
$$

which is the total demand for heat during period $t$. The degree-day unit is the difference in degrees between the mean daily outside temperature and the desired inside temperature, say $18^{\circ} \mathrm{C}$. The heat-transfer coefficient will be affected by the amount and quality of insulation used in the building and can be treated as a decision variable in the system. For simplicity, however, $h$ will be treated as a parameter specific to the building being considered.

If (3) exceeds (2) plus the available heat in storage during any period, then the heat deficit must be made up by an auxiliary heating system. Electric resistance heating is usually chosen for this purpose, because it has low capital costs which complement the high capital costs of the solar heating system. The heat in period $t$ generated by the electric system is denoted by

$$
c E_{t}
$$

where $E_{t}$ is kilowatt hours of electricity and $c$ converts the kilowatt hours to Btu's of heat. The electrically produced heat can also be pumped into storage which makes the model more general as pointed out in Section V. The capital cost of the electric system is assumed to be the minimum cost of ensuring that the building can be heated to a comfortable temperature under the most extreme weather conditions using only electricity. This would be the same cost that an all-electric, nonsolar building would incur. Therefore, the auxiliary system capital costs need not enter into the analysis, since this cost would be incurred regardless of whether solar heating is used. ${ }^{3}$ To ensure that electricity is the best auxiliary source, the solar/electric system can be compared with other auxiliary sources (e.g., solar/natural gas, solar/oil, solar/wood). The model used here can evaluate the cost of these other systems by appropriate modification to (4). In making the comparison, the capital costs of all the auxiliary systems must be taken into account as well, by adding them to the costs determined by the model.

The total available heat during period $t$ is the sum of the electric heat, the collector heat and the available storage heat from period $t-1$. Subtracting both the demand for heat in period $t$ and the heat lost to overflow because of insufficient storage capacity from the available heat yields the excess supply of heat in period $t$. This excess supply is given by the following recursive equation which combines (2), (3), and (4). ${ }^{4}$

3. This is the standard approach taken to determine a least-cost system $[4 ; 9 ; 14 ; 15 ; 26]$ and the model is appropriate for new buildings or for adding on to existing buildings.

4. This recursive equation represents a modification in an equation formulated in an earlier paper by Hamlen, et. al. [12]. The equation in [12] was designed for computational efficiency while (5) is generalized to capture the complete economic aspects of the problem. 


$$
X_{t}=s_{t} b A+c E_{t}+e X_{t-1}-k_{t} h-L_{t}
$$

The storage efficiency is captured by $e$ which can be thought of as an efficiency rating. Clearly $0 \leq e \leq 1$. Equation (5) can be solved to obtain

$$
X_{t}=\sum_{j=0}^{t-1} e^{j}\left[s_{t-j} b A+c E_{t-j}-k_{t-j} h-L_{t-j}\right]
$$

Equation (6) illustrates the role of the storage efficiency. If $e=0$, then $X_{t}$ is simply supply minus demand generated in period $t$. There is no storage capability in this case. If $e=1$, then all of the excess supply from past periods is available in the current period. But suppose $e=$ $1 / 2$, then in period $t=5,1 / 32$ of the excess supply of heat from period 1 is available in period 5. The efficiency rating is assumed to be fixed in the model and less than one; however, $e$ could be treated as a variable without difficulty.

\section{The Solar Energy-Public Utility Interface}

There are, to this point, four decision variables that the building owner must combine to determine the minimum cost heating system. For given efficiency ratings of the collector and storage, the three decision variables in the solar heating system are the collector area, the storage capacity, and the heat lost to overflow. For the auxiliary system, the decision variable is the kilowatt hours of electricity. The collector area and storage capability are scalar valued variables, since only one size is chosen over the entire planning horizon. The electricity and heat loss, however, are vectors where the components are the electricity and heat loss for a particular period within the planning horizon. Thus, the decision variables are given by $A, S$, $L=\left(L_{1}, \cdots, L_{n}\right)$ and $E=\left(E_{1}, \cdots, E_{n}\right)$.

The heating problem is complicated by uncertain climatic conditions: the demand for heat depends on the outside temperature and the supply of heat depends on the available solar radiation. An important consideration is whether the decision variables are ex ante or ex post; that is are they chosen before or after the values of the random variables, temperature $\left(k_{t}\right)$ and radiation $\left(s_{t}\right)$, are known. Since the collector and storage cannot readily be changed once they are installed, they are ex ante variables. However, electricity may be ex ante or ex post depending on availability. Typically, residential, commercial and many industrial users of electricity set their thermostats at some temperature, and however many kilowatt hours of electricity are required to maintain that temperature are supplied by the public utility. This makes electricity an ex post variable. ${ }^{5}$ However, it also thrusts the entire burden of uncertainty onto the utility. Demand for electricity will fluctuate with weather conditions, and if the utility is obligated to supply this electricity always, enormous generating capacity must be available to meet the extreme weather conditions. This generating capacity is then idle during less severe weather.

The addition of solar heated buildings to the public utility system can exacerbate the utility's problem. The solar user's peak demand for electricity will occur during cold, cloudy weather when the collector and storage provide little heat. But this peak demand will be the same as for a comparable nonsolar building. Therefore, if the utility must supply all the electricity that is demanded, then the same generating capacity must be available for solar users

5. This is the implicit assumption used in $[4 ; 9 ; 14 ; 19 ; 26]$. 
as is available for nonsolar users. And generating capacity represents a large part of the cost of supplying electricity. Hence, the solar user will demand less kilowatt hours of electricity than the nonsolar user, but both will have the same peak demand. Solar users, in other words, exhibit low load factors. The result is that widely used pricing methods, such as declining block tariffs, may generate insufficient revenues to cover the costs of serving solar users. This is unsatisfactory from the utility's viewpoint, and from nonsolar users who would have to subsidize the solar users. Some suggested remedies for this problem have included: 1) fixed demand charges for solar users only, and 2) increases in the early blocks of declining block tariffs. ${ }^{6}$ Neither of these approaches really addresses the key issue, however, and this is that the burden of uncertainty continues to lie with the utility. In addition, these suggested remedies may have a dampening effect on the widespread adoption of solar energy.

The model developed here represents a way of transferring at least some of the uncertainty onto the consumers. Each consumer is the best judge of how much uncertainty he or she is willing to accept with regards to maintaining a warm building. By removing the uncertainty from the utility, the need to construct very large generation capacity, which may often be idle, is reduced. To accomplish this transfer of uncertainty, electricity is treated as an interruptible service whereby its supply can be curtailed under certain conditions. Among industrial consumers, interruptible service is not uncommon in the U.S., and these consumers may enjoy lower rates in return for the greater uncertainty of supply. In Europe, some consumers subscribe to particular levels of capacity and their consumption is curtailed if they exceed this level. In addition, the 1979 National Energy Act requires state regulatory authorities to consider the applicability of interruptible rates while at the same time declining block rates, if not cost justified, are to be discontinued. Feldman and Anderson [9] have also suggested that interruptible rates appear to be an attractive alternative for solar users.

Interruptible service can be added to the present model in a number of ways.' One way is to stipulate that the consumer cannot demand more than $F$ kilowatts of electricity at any time, where $F$ is then a consumer capacity constraint. This capacity can be thought of as a fuse size; when the consumer attempts to use more electricity than $F$ allows, the fuse blows. Some advantages of this system, which are detailed in [18], are that the utility can better plan for capacity requirements, since the uncertainty of peak demand is reduced. Also, the utility would not have to treat solar users differently than nonsolar users. $F$ is a fifth decision variable for the building owner, and larger values of $F$ will mean greater reliability of warmth, but at greater cost to the owner. This reliability/cost tradeoff is discussed further in the next section.

\section{The Cost Minimization Model}

Let $p$ be the discounted equivalent annual cost per unit area of $A, C(S)$ be the discounted annual cost of storage heat capacity, $w$ be the price of a kilowatt hour of electricity, and let $r$ be the price of a kilowatt of $F$. The latter two prices, $w$ and $r$, can be thought of as an energy

6. Lorsch [15] makes these suggestions and he also points out that because of rate structures like declining block tariffs, the monetary savings enjoyed by a solar homeowner will be less than proportional to the auxiliary energy saved.

7. Recent papers that deal with different forms of interruptible service for public utilities include Marchand [16], Panzar and Sibley [18], and Tschirhart and Jen [25]. 
charge and a demand charge for electricity respectively. Total cost of the heating system in one year consisting of $n$ equal periods is then ${ }^{8}$

$$
p A+C(S)+w \sum_{t=1}^{n} E_{t}+r F
$$

Note that there is no risk involved in this expression. The optimum values of the collector size, storage, fuse size, and electricity, denoted $A^{*}, S^{*}, F^{*}$, and $E^{*}$, are chosen and substituted into (7) to yield total cost. The risk enters because at $A^{*}, S^{*}, F^{*}$, and $E^{*}$, there may be a positive probability that the building is not adequately heated. To limit this risk to acceptable levels, chance constraints are used which require that during each period $t$, the demand for heat must not exceed the supply of heat with a specified probability.

Using (6), the constraint for period $t$ is written as

$$
\operatorname{Pr}\left\{X_{t} \leq 0\right\} \leq \rho_{t}
$$

where $\operatorname{Pr}$ denotes probability and $\rho_{t}$ is a non-negative number less than one. The solution to the problem provides the owner with a solar heating system and auxiliary backup that ensure $18^{\circ} \mathrm{C}$ with a probability of $1-\rho_{t}$ on day $t$. The term $1-\rho_{t}$ is a measure of the risk level that the owner is willing to accept. The parameter $\rho_{t}$ is chosen based on the costs, pecuniary or otherwise, if not maintaining an inside temperature of $18^{\circ} \mathrm{C}$. The higher these costs, the nearer $\rho_{t}$ is to zero. The objective function provides the building owner with the cost of a heating system that will satisfy heating needs with a reliability level of $1-\rho_{t}$ in each period $t$.

Risk adversion in this model refers to the owner's attitude toward not having adequate heat. Decreasing the value of $\rho_{t}$ allows more risk in return for lower costs. Solving a series of problems for various values of $\rho_{t}$ permits the owner to achieve the optimum risk-cost tradeoff. As suggested earlier, this decision is best left to the individual consumers, as opposed to the common practice of having the public utility strive for $100 \%$ reliability regardless of the cost. When allowed to choose between risk and costs, consumers may very well opt for more risk at lower costs relative to the historical tradition in the U.S. where risk is extremely small. ${ }^{9}$ If the fuse system is extended to both solar and nonsolar consumers, then both have the option of trading off risk for cost. For consumers with identical heating requirements, a nonsolar consumer must choose a larger fuse than a solar consumer to achieve the same reliability level. Thus, solar consumers can save on both energy and demand charges, while at the same time, they do not place any greater burden on the utility than do nonsolar consumers. The success of this system, or any other form of interruptible service in reducing the burden of uncertainty to the utility will depend heavily on the enforcement of contracts between the utility and the consumers regarding fuse size. If consumers are allowed to exchange fuses at will, then the utility's ability to plan for future capacity and to manage loads is limited, and the advantages of interruptible service are lost. Thus, long-term contracts are necessary to procure these advantages. To ensure that the electricity used does not exceed the fuse size, $n$ constraints are needed, one for each period, as given by (9).

$$
E_{t} \leqq F
$$

The excess supply of heat in any period that is to be used in subsequent periods is

8. $C(S)$ is assumed to be continuously differentiable with $C^{\prime}(S)>0$.

9. Telson [24] discusses the possibility that loss-of-load probabilities in the U.S. may be too high from an economic standpoint. Studies typically place the reliability of electric service in excess of $99.99 \%$. 
bounded by the storage capacity. This calls for another set of constraints which ensure that the heat carried from one period to the next is not greater than that which the storage system can absorb. These constraints, like the previous constraints of (8), involve the random variables; however, unlike the previous constraints, they represent a technical restriction on the model. If $X_{t}>S$, the model is failing to accurately represent the technical aspects of the heating system. For $X_{t}$ to exceed $S$ is impossible. Therefore, these constraints must take the form

$$
\operatorname{Pr}\left\{X_{t} \leq S\right\}=1
$$

for each period $t$.

The role of heat loss, $L_{t}$, now becomes more vivid. Constraint (10) can be met by using a very large storage capacity. But storage is costly and the solution may call for a smaller capacity with positive heat losses in some periods. Alternatively, (10) can be met by using a small collector, but this may result in not taking full economic advantage of solar radiation. The solution may call for a larger collector with positive heat losses in some periods to satisfy (10).

The length of the time period $t$ is crucial owing to the characteristics of incoming solar radiation. For each time period there are two random variables, and the parameters in the distributions of these variables depend on the length of the specified period. Selecting a relatively long period such as a week $(n=52)$, a month $(n=12)$, or even a year $(n=1)$ eases difficulties in data collection and computation. However, the following discussion illustrates that a much shorter time period is required.

Hamlen, Hamlen, and Tschirhart [13] present results of a model used by Hamlen and Hamlen [11] to predict local levels of incoming solar radiation. The results indicate that the daily radiation levels display geometric Brownian motion. This implies that the probabilities of daily incoming solar radiation either increasing or decreasing by 1 percentage unit are equal and independent of the level of radiation. Let $s_{t}$ and $s_{t+1}$ be incoming radiation on successive days; then the elements of the sequence $z_{1}, z_{2}, \cdots$, where $z_{t+1}=s_{t+1} / s_{t}$ are independent and identically log-normally distributed random variables. As shown in [13], this implies that the distribution of any $s_{t}$ which follows $t$ days after an initial amount $s_{0}$ is log-normally distributed and dependent on the four parameters, $t, s_{0}$, and the mean and variance of the $z$ terms. Specifically, the mean and variance of $s_{t}$ are given by (11) and (12) respectively.

$$
\begin{aligned}
\mu_{s_{t}} & =\exp \left[t \mu_{z}+\ln s_{0}+1 / 2 t \sigma_{z}{ }^{2}\right] \\
\sigma_{s_{t}}{ }^{2} & =\mu_{s_{t}}{ }^{2}\left[\exp \left[t \sigma_{z}{ }^{2}\right]-1\right]
\end{aligned}
$$

A more comprehensive derivation of (11) and (12) appears in Appendix B.

These results show that solar radiation for each day has a distinct distribution; therefore, the duration of the time period should not exceed one day. If the time period exceeds one day then joint distributions of daily solar radiation would be needed, and this would unnecessarily complicate matters. One day is a relatively short time period, since a year long problem requires 365 of the constraints given by (8), (9), and (10). Nevertheless, the stochastic nature of solar radiation dictates this approach. The alternative would be to use historical averages for the solar radiation. But historical averages, as Tybout and Löf $[26,76]$ point out,“... fail to reveal the patterns of sunny and cloudy weather that follow from serial correlation of climate over time. It is essential that the latter be taken into account in the opti- 
mization of the collector to storage ratio." The approach of Tybout and Löf and Ben-David et al. is to build a simulation model and use actual hour-by-hour weather observations from what was judged to be a "typical year." The optimization approach more fully captures the probabilistic character of the solution, and allows for greater clarity in the presentation of the economic tradeoffs involved. In application, the number of constraints may be reduced considerably by eliminating the warmer months when heat is not needed. Of course, the months that are eliminated depend on the geographic location of the building. The total number of days used henceforth in the model is denoted by $T$.

For convenience, the chance constraints can be written in equivalent riskless forms. ${ }^{10}$ Let $G_{t}$ be the distribution function of the standardized random variable $\left(X_{t}-\bar{X}_{t}\right) / \sigma_{X_{t}}$ where $\bar{X}_{t}$ and $\sigma_{X_{t}}$ are the mean and standard deviation of $X_{t}{ }^{11}$ The constraints given by (8) and (10) can be then written as

$$
G_{t}\left(-\bar{X}_{t} / \sigma_{X_{t}}\right) \leqq \rho_{t} \quad \text { and } \quad G_{t}\left(\left(S-\bar{X}_{t}\right) / \sigma_{X_{t}}\right)=1
$$

respectively. In turn these become $\left(8^{\prime}\right)$ and $\left(10^{\prime}\right)$.

$$
\begin{gathered}
\bar{X}_{t} / \sigma_{X_{t}} \geqq-N_{t}\left(\rho_{t}\right) \\
\left(S-\bar{X}_{t}\right) / \sigma_{X_{t}}=N_{t}(1)
\end{gathered}
$$

$N_{t}$ is the inverse function of $G_{t}$ and can be thought of as the number of standard deviations from the mean necessary to reduce the area in the lower tail of the distribution to $\rho_{t}$ for $\left(8^{\prime}\right)$, and to effectively eliminate the upper tail of the distribution for $\left(10^{\prime}\right)$. In $\left(8^{\prime}\right), N_{t}$ can also be interpreted as the minimum probabilistic requirement for the standardized mean excess supply; in $\left(10^{\prime}\right) N_{t}$ can also be interpreted as the standardized mean excess capacity of storage. ${ }^{12}$

The problem of minimizing cost subject to the constraints is written as

$$
\begin{gathered}
\text { maximize }-p A-C(S)-w \sum_{t=1}^{T} E_{t}-r F \\
\text { subject to } f_{t}\left(A, E_{t}, L_{t}\right)=\bar{X}_{t}+\sigma_{X_{t}} N_{t}\left(\rho_{t}\right) \geqq 0 \\
\qquad q_{t}\left(E_{t}, F\right)=F-E_{t} \geqq 0 \\
g_{t}\left(A, E_{t}, L_{t}, S\right)=S-\bar{X}_{t}-\sigma_{X_{t}} N_{t}(1)=0
\end{gathered}
$$

for all $t$. The conditions for which both the objective function and constraints are concave in $A, E, L, F$, and $S$ are discussed in Appendix A, and the results in Appendix A can also be used to show that the rank condition on the mixed constraints is satisfied. Therefore, the Kuhn-Tucker conditions given by (14)-(29) are necessary and sufficient for a maximum at $\left(A^{*}, E^{*}, L^{*}, F^{*}, S^{*}, \lambda^{*}, \gamma^{*}, \delta^{*}\right)$ where $\lambda^{*}=\left(\lambda_{1}{ }^{*}, \cdots, \lambda_{T}{ }^{*}\right)$ is the vector of multipliers for the constraints of $f_{t}, \gamma^{*}=\left(\gamma_{1}^{*}, \cdots, \gamma_{T}^{*}\right)$ is the vector of multipliers for the constraints on $g_{v}$, and $\delta^{*}=\left(\delta_{1}^{*}, \cdots, \delta_{T}^{*}\right)$ is the vector of multipliers for the constraints on $q_{t}$.

$$
A: \quad A^{*}\left[-p+\sum_{j=1}^{T}\left[\lambda_{j}^{*} \partial f_{j} / \partial A+\gamma_{j}^{*} \partial g_{j} / \partial A\right]\right]=0
$$

10. See Charnes and Cooper [6] for a presentation of this methodology.

11. The mean and standard deviation of $X_{t}$ are defined in Appendix A.

12. The reader can reinforce his understanding of this concept by noting that $N_{t}\left(\rho_{t}\right)$ is negative and a less negative value for $N_{t}\left(\rho_{t}\right)$ implies a less restrictive constraint in (8). See Meyer [17] and Tschirhart and Jen [25] for other uses of this method. 


$$
\begin{aligned}
& -p+\sum_{j=1}^{T}\left[\lambda_{j}^{*} \partial f_{j} / \partial A+\gamma_{j}^{*} \partial g_{j} / \partial A\right] \leqq 0 \\
& E_{i}: \quad E_{t}^{*}\left[-w+\sum_{j=t}^{T}\left[\lambda_{j}^{*} \partial f_{j} / \partial E_{t}+\gamma_{j}^{*} \partial g_{j} / \partial E_{t}\right]-\delta_{t}^{*}\right]=0 \\
& -w+\sum_{j=t}^{T}\left[\lambda_{j}^{*} \partial f_{j} / \partial E_{t}+\gamma_{j}^{*} \partial g_{j} / \partial E_{t}\right]-\delta_{t}^{*} \leqq 0 \\
& S: \quad S^{*}\left[-C^{\prime}(S)+\sum_{j=1}^{T} \gamma_{j}^{*} \partial g_{j} / \partial S\right]=0 \\
& -C^{\prime}(S)+\sum_{j=1}^{T} \gamma_{j}^{*} \partial g_{j} / \partial S \leqq 0 \\
& L_{t}: L_{t}^{*}\left[\sum_{j=1}^{T}\left[\lambda_{j}^{*} \partial f_{j} / \partial L_{t}+\gamma_{j}^{*} \partial g_{j} / \partial L_{t}\right]\right]=0 \\
& \sum_{j=1}^{T}\left[\lambda_{j}^{*} \partial f_{j} / \partial L_{t}+\gamma_{j}^{*} \partial g_{j} / \partial L_{t}\right] \leqq 0 \\
& F: F^{*}\left[-r+\sum_{j=1}^{T} \delta_{j}^{*}\right]=0 \\
& -r+\sum_{j=1}^{T} \delta_{j}^{*} \leqq 0 \\
& \lambda_{t}: \lambda_{t}^{*} f_{t}\left(A^{*}, E_{t}^{*}, L_{t}^{*}\right)=0 \\
& f_{t}\left(A^{*}, E_{t}^{*}, L_{t}^{*}\right) \geqq 0 \\
& \delta_{t}: \delta_{t}^{*} q_{t}\left(E_{t}^{*}, F_{t}^{*}\right)=0 \\
& q_{t}\left(E_{t}^{*}, F_{t}^{*}\right) \geqq 0 \\
& \gamma_{t}: g_{t}\left(A^{*}, E_{t}^{*}, L_{t}^{*}, S^{*}\right)=0 \\
& A^{*}, E_{t}^{*}, L_{t}^{*}, S^{*}, \lambda_{t}^{*}, \delta_{t}^{*}, \geqq 0
\end{aligned}
$$

Conditions (16), (17), (20), (21), and (24) through (29) are for all $t$.

\section{Results of the Model}

To examine these conditions, the multipliers $\lambda, \gamma$, and $\delta$ can be interpreted as the shadow prices of heating system reliability, storage capacity feasibility, and fuse size feasibility respectively. ${ }^{13}$ A positive shadow price for reliability in period $t, \lambda_{t}^{*}>0$, implies that $f_{t}\left(A^{*}, E_{t}^{*}\right.$, $\left.L_{t}^{*}\right)=0$ from (24). In this case, heating system reliability is achieved exactly; the probability that the building is colder than the desired temperature in period $t$ is $\rho_{r}$. Reliability is a binding constraint on the system, and if this constraint is relaxed slightly ( $\rho_{t}$ increased) a marginal

13. The shadow prices can be used to allocate the cost of the heating system over the various time periods. This would be appropriate if there were different building occupants such as in a sports arena. If the model was applied to the production of solar electricity by a public utility, the multipliers could allocate costs among peak and offpeak consumers. In this case, the reliability constraints are similar to those used by utilities to achieve a particular level of loss-of-load probability (see Anderson [1] for this methodology). More detail on the use of the shadow prices is available from the authors. 
cost savings of $\lambda_{t}^{*}$ could be realized. Note that a positive $\lambda_{t}^{*}$ does not imply that the desired temperature is exactly met. Inside temperature is dependent on the randomness of both outside temperature and solar radiation, and this inside temperature can be achieved only in a probabilistic sense. If $f_{t}\left(A^{*}, E_{t}^{*}, L_{t}^{*}\right)>0$, then heating system reliability exceeds the minimum requirements and $\lambda_{t}^{*}=0$ from (24). Relaxing the constraint slightly would not result in a cost savings.

A positive shadow price for storage capacity feasibility implies that a slight relaxation of the constraint in (28) will permit lower costs of $\gamma_{t}^{*}$ in period $t$. By relaxing the equality constraint, the possibility that the heat pumped into storage exceeds capacity is allowed. This, in turn, would mean less need for heat overflow and more heat available for the future, thereby requiring less future kilowatt hours of electricity and a smaller collector. This is how costs would be saved. However, this relaxation is infeasible, since storage cannot hold more heat than capacity allows. The shadow price indicates the costs involved with maintaining a feasible system and allowing for overflow.

When $\delta_{t}^{*}$ is positive, then the kilowatt hours of electricity used on day $t$ are equal to the fuse size. This is the maximum possible use of electricity. The positive shadow price indicates that if a slightly larger fuse were used on day $t$, or if slightly more electricity in excess of the fuse size could be used on day $t$, then a cost savings of about $\delta_{t}^{*}$ would be achieved. But this violates system feasibility, and $\delta_{t}^{*}$ is the cost of maintaining feasibility. On any day $t$ where the electricity used is less than the fuse size, $\delta_{t}{ }^{*}=0$ and the fuse size is a nonbinding constraint.

For $A^{*}>0,(15)$ is satisfied by equality so that the marginal cost of increasing the collector, $p$, must be equal to the sum over all days of: a) the shadow price of reliability times the marginal reliability from a change in collector size, plus b) the shadow price of storage capacity feasibility times the marginal excess storage capacity from a change in collector size. From Appendix $\mathrm{A}, \partial g_{t} / \partial A<0$ for all $t$ so that an increase in collector size implies a decrease in the mean excess storage. Thus, the size of the collector is increased until its marginal cost is balanced with the positive effects a larger collector has on reliability and the negative effects a larger collector has on storage feasibility.

For the kilowatt hours of electricity, a similar interpretation holds except that the electricity used in period $t$ affects only the reliabilities and storage in periods beyond $t$. Also, the shadow price on the fuse size enters into (16) and (17). Therefore, electricity in period $t$ is chosen so that its marginal cost is balanced against its effects on the current and all future reliabilities and future storage and fuse size limitations.

Equations (18) and (19) indicate that if storage is used and $S^{*}>0$, then

$$
C^{\prime}(S)=\sum_{j=1}^{T} \gamma_{j}^{*} \partial g_{j} / \partial S
$$

From Appendix A, $\partial g_{j} / \partial S=1$ which yields

$$
C^{\prime}(S)=\sum_{j=1}^{T} \gamma_{j}^{*}
$$

Therefore, the sum of the shadow prices on capacity feasibility is equal to the marginal cost of capacity. Moreover, at least one $\gamma_{t}^{*}$ must be positive indicating that in at least one period, capacity limits the amount of heat available for future periods.

Again, using Appendix A, (20) can be written as 


$$
L_{t}^{*}\left[\sum_{j=t}^{T} e^{j-t}\left[\gamma_{j}^{*}-\lambda_{j}^{*}\right]\right]=0 .
$$

The bracketed term is the summation of the differences between the shadow prices of storage and reliability where each difference is weighted by a decay factor determined by storage efficiency. If there is a heat loss to overflow in period $t$, then the bracketed term equals zero. Heat lost in period $t$ has a negative effect on future reliabilities and a positive effect on meeting future storage constraints. Thus, if $L_{t}^{*}>0$, these negative and positive effects must balance. Alternatively, when the negative effect on future reliabilities outweigh the positive effects on storage, that is

$$
\sum_{j=t}^{T} e^{j-t}\left[\gamma_{j}^{*}-\lambda_{j}^{*}\right]<0,
$$

then no heat is lost to overflow. Also, if $L_{t}{ }^{*}>0$, then from (31) $\lambda_{t-1}{ }^{*} \geqq \gamma_{t-1}{ }^{*}$. This says that an overflow of heat today must mean that the shadow price of yesterday's reliability is at least as great as the shadow price of yesterday's feasibility. Roughly, the size of yesterday's storage is relatively unrestrictive if heat is being wasted today.

Equations (22) and (23) indicate that if electricity is used so that $F^{*}>0$, then

$$
r=\sum_{j=1}^{T} \delta_{j}^{*}
$$

The sum of the shadow prices on fuse size feasibility is equal to the marginal cost of the fuse. Since at least one $\delta_{j}^{*}$ must be positive to satisfy (32), the implication is that if electricity is used at all then the fuse size will be binding on at least one day.

To illustrate the interaction between the variables, assume initially that the fuse size is not binding in periods $t$ and $t+1$. Hence, $\delta_{t}{ }^{*}=\delta_{t+1}{ }^{*}=0$ and combining (16) and (17) and results from Appendix A yields

$$
\begin{gathered}
E_{t}^{*}\left[-w+\sum_{j=t}^{T} c e^{j-t}\left[\lambda_{j}^{*}-\gamma_{j}^{*}\right]\right]=0 \\
-w+\sum_{j=t}^{T} c e^{j-t}\left[\lambda_{j}^{*}-\gamma_{j}^{*}\right] \leqq 0 .
\end{gathered}
$$

These expressions along with (31) can be used to show that in periods where $f_{t}\left(A^{*}, E_{t}^{*}, L_{t}^{*}\right)$ $>0$ (that is, when the heating system reliability exceeds the minimum requirements), electricity is not used. This is intuitively appealing because with a nonbinding fuse size there is no reason to use costly electricity to increase heat output beyond the minimum requirements. One might argue that electricity could heat storage to ensure adequate reliability in subsequent periods. However, provided there is some heat loss from storage $(e<1)$, the heat needed in subsequent periods is better obtained from electricity in subsequent periods, since the heat generated from this latter electricity is not subject to storage losses.

To show this result, consider two cases: first, the case where storage is binding in period $t$ and $L_{t}>0$. Then from (31)

$$
\sum_{j=t}^{T} e^{j-t}\left[\gamma_{j}^{*}-\lambda_{j}^{*}\right]=0
$$

and using (33) and (34) this implies that $E_{t}^{*}=0$. Heat lost to overflow and electricity can never both be positive. Second, consider the case where storage is not binding so that the 
shadow price is zero. Therefore, $\gamma_{t}^{*}=0$ and from $f_{t}\left(A^{*}, E_{t}^{*}, L_{t}^{*}\right)>0, \lambda_{t}{ }^{*}=0$. Substituting these values into (34) yields

$$
-w+\sum_{j=t+1}^{T} c e^{j-t}\left[\lambda_{j}^{*}-\gamma_{j}^{*}\right] \leqq 0
$$

which can be rewritten as

$$
-w+e \sum_{j=t+1}^{T} c e^{j-t-1}\left[\lambda_{j}^{*}-\gamma_{j}^{*}\right] \leqq 0 .
$$

But for period $t+1,(34)$ is written as

$$
-w+\sum_{j=t+1}^{T} c e^{j-t-1}\left[\lambda_{j}^{*}-\gamma_{j}^{*}\right] \leqq 0 .
$$

If (36) is satisfied by either equality or inequality, (35) must be satisfied by the inequality and $E_{t}{ }^{*}=0$ from (33). This second case represents the day where both electricity and heat lost to overflow are zero; this occurs if solar radiation alone could meet the reliability requirements but not generate sufficient heat to fill storage. That $e<1$ is the key to this second case, since if $e=1$ the heating requirements on day $t+1$ could be met equally well by electricity on day $t+1$ or electricity on day $t$ which is pumped into storage.

The assumption that the fuse size is not binding in periods $t$ and $t+1$ is now relaxed so that $\delta_{t}{ }^{*} \geqq 0$ and $\delta_{t+1}{ }^{*} \geqq 0$. This alters the previous expressions as follows: $\delta_{t}^{*}$ is subtracted from the left-hand side of (33), (34), and (35) and $\delta_{t+1}{ }^{*}$ is subtracted from the left-hand side of (36). The implications are that in contrast to the previous result, electricity may be used even if the heating system reliability exceeds the minimum requirements. Again, consider the two cases: first, where storage is binding in period $t$ and $L_{t}>0$. Here, (31), (33), and (34) are still used to show that $E_{t}{ }^{*}=0$. This result does not change; electricity is never used if heat overflow losses occur. In the second case, however, where storage is not binding, electricity may be used in period $t$ beyond that which is required to maintain $18^{\circ} \mathrm{C}$. This electricity is used to heat storage which can then be drawn upon for heat in period $t+1$. The reason for the change from the previous result is that the fuse size is now binding and it may not be possible to draw enough electricity on day $t+1$ to meet the heating requirements. Therefore, electricity on day $t$ is used via storage in spite of the fact that some of the heat from this electricity will be lost to storage inefficiency $(e<1)$.

The mathematics of this second case derive from (35) and (36) with $\delta_{t}{ }^{*}$ and $\delta_{t+1}{ }^{*}$ in place. If (36) is satisfied by equality (a fortiori by inequality) then substitution into (35) yields

$$
w(e-1)+e \delta_{t+1}{ }^{*}-\delta_{t}{ }^{*} \leqq 0 .
$$

A sufficient condition for (37) to be satisfied by the inequality and $E_{t}=0$ is that $e \delta_{r+1}{ }^{*} \leqq \delta_{t}^{*}$. The interpretation is that electricity should not be used in period $t$ to heat storage if the fuse size is more restrictive in period $t$ than in period $t+1$ when the latter period is weighted by storage efficiency. Alternatively, electricity should be used in period $t$ to heat storage in order to supply heat for period $t+1$ only if the fuse size will not allow adequate electricity in period $t+1$ to meet the heating requirements.

Until now the possibility that the price per kilowatt hour of electricity may vary within each period has not been considered. Time-of-day or peak-load pricing is becoming more commonplace, however, as a means of more efficiently utilizing energy resources by allowing 
prices to closely track marginal cost. ${ }^{14}$ Peak-load pricing has some very interesting implications for solar energy use which can be illustrated with a few straightforward alterations to the model herein. First, $E_{t}$ must be separated into two parts: an off-peak component and a peak component. More than two components could also be considered but for simplicity only two are used here. Second, the price $w$ must be separated into an off-peak price and a peak price. And third, $X_{t}$ must be separated into an off-peak and peak component where the nighttime component does not contain solar radiation as a heat source. With these modifications and minor notational changes, the model is generalized to cover a peak-load pricing rate structure. Details of these changes can be obtained from the authors, and only the implications are discussed here.

If the off-peak price for electricity is significantly lower than the peak price, then heating storage with electricity in the off-peak period may be economically attractive. ${ }^{15}$ With a larger storage capacity, the solar user could ensure that heat in storage obtained from either radiation or off-peak electricity is adequate to get through the peak period without using electricity. The greater the discrepancy between peak and off-peak price, the greater the incentive to use a larger storage to avoid the peak price. In a sense, solar heating is now forced to compete with inexpensive off-peak electricity, and this makes solar heat a less competitive alternative.

Some authors [9] have suggested that solar users might actually be barred from using peak electricity. If such a practice were to become very widespread, then off-peak demand could conceivably increase to a point where there would be little or no difference between demand in the two periods. The extent to which this might occur depends on whether a firm or shifting peak situation exists (see Steiner [23]).

To determine the competitiveness of solar heating verus conventional, the possibility of heating storage with off-peak electricity underscores an important point. The solar heating system should not be compared with a conventional electric heating system; instead, the solar heating system should be compared with a conventional electric heating system that has a storage component. Asbury and Mueller contend that "... most of the electricity supply savings claimed for solar energy systems stem from the storage, rather than the solar, component of the systems" $[2,446]$. The model developed above can be used to address this issue. If their contention is accurate for a particular geographic area, then the solution will yield $A^{*}=0$ and $S^{*}>0$. Thus, the model encompasses three systems: 1) solar with auxiliary heat, 2) all electric, and 3) all electric with storage.

\section{Conclusion}

In this paper, a new approach to determining the economically efficient use of solar energy has been presented. The approach has several significant advantages. First, the randomness

14. The 1979 National Energy Act [27] directs state regulatory agencies to consider the applicability of timeof-day rates and seasonal rates. See Wenders and Taylor [28] for an analysis of nonsolar seasonal pricing. Solar heating will have varying influences on summer-peaking versus winter-peaking utilities. Most utilities are now or will be summer peaking. In this case, solar heating may result in even lower load factors, since the solar consumers' peak demand is in the summer [9]. Thus a form of interruptible service appears increasingly attractive. Analysis of seasonal demand variations is beyond the scope of this paper.

15. If $w_{p}$ and $w_{o p}$ are the peak and off-peak prices respectively, then a necessary condition for using off-peak electricity to heat storage is $w_{o p}<e w_{p}$. The off-peak price must be less than the peak price when the latter is weighted by the storage efficiency. 
of both the demand for heat and the supply of solar radiation have been incorporated. Second, the particular nature of the distribution for solar radiation used, that is, the geometric Brownian distribution, is compatible with the chance-constrained methodology. Third, optimization as opposed to simulation techniques are utilized, although no more data is required. Fourth, the model allows for a transfer of uncertainty from utilities to consumers, thereby making the adoption of solar energy more palatable to the former group.

The importance of developing techniques that allow for efficient use of solar energy cannot be overstated, since the sun is becoming increasingly attractive as an energy source in light of dwindling stocks of fossil fuels and the pollution associated with their use. This is not to say that solar energy is not without problems or that a solar economy is a near term possibility. ${ }^{16}$ However, the U.S. is committed to a program of solar energy growth as exemplified by the National Energy Act and by recent presidential addresses. To be successful, the program must ensure that solar energy is used in an economically efficient manner and that a careful interfacing with existing technologies is accomplished.

\section{Appendix A}

The mean of $X_{t}$ as given by (6) is

$$
\bar{X}_{t}=\sum_{j=0}^{t-1} e^{j}\left[\mu_{s_{t-j}} b A+c E_{t-j}-\mu_{k_{t-j}} h-L_{t-j}\right]
$$

where $\mu_{s_{i-j}}$ is the mean solar radiation from (11) and $\mu_{k_{t-j}}$ is the mean degree-day unit. The degree-day unit has been found to have a normal distribution [11]. ${ }^{17}$ The daily amounts of solar radiation are independently distributed as are the daily degree-day units. Moreover, solar radiation is independent of temperature based on both theoretic and empirical investigations. Therefore, the standard deviation of $X_{t}$ is

$$
\sigma_{X_{t}}=\left\{\sum_{j=0}^{t-1} e^{2 j}\left[\sigma_{s_{t-j}}{ }^{2} b^{2} A^{2}+\sigma_{k_{t-j}}{ }^{2} h^{2}\right]\right\}^{1 / 2}
$$

where $\sigma_{s_{t-j}}{ }^{2}$ is the variance of solar radiation from (12) and $\sigma_{k_{t-j}}$ is the variance of the degree-day unit.

From (41) and (42), the following partials are easily derived.

$$
\left.\begin{array}{ll}
\partial \bar{X}_{t} / \partial A=\sum_{j=0}^{t-1} e^{j} \mu_{s_{t-j}} b>0 & \partial \sigma_{X_{t}} / \partial A=\left(1 / \sigma_{X_{t}}\right) \sum_{j=0}^{t-1} e^{2 j}{\sigma_{s_{t-j}}}^{2} b^{2} A>0 \\
\partial \bar{X}_{t+j} / \partial E_{t}=e^{j} t>0 & \partial \sigma_{X_{t+j}} / \partial E_{t}=0 \\
\partial \bar{X}_{t+j} / \partial L_{t}=-e^{j}<0 & \partial \sigma_{X_{t+j}} / \partial L_{t}=0
\end{array}\right\} \begin{aligned}
& j=0, \cdots, T-t \\
& t=1, \cdots, T
\end{aligned}
$$

Substituting (41) and (42) into the constraints of problem (13) and using the above partials, the following terms used in the text are obtained.

16. Georgescu-Roegen [10] has taken a pessimistic view of solar energy. In short, he argues that solar collectors cannot produce more collectors from the energy they collect. Solar is therefore " ... a parasite of the current technology. And, like any parasite, it could not survive its host" [10, 1053]. He admits, however, that more efficient technologies may radically change his conclusions. It is not unreasonable to expect radical changes given that only very recently has solar energy been emphasized on a grand scale. Also, his arguments would seem inappropriate for passive solar heating technologies. This paper has dwelt on active systems, but could be suitably modified to passive systems.

17. The limits for a normal distribution are $\pm \infty$ : therefore, some modification of (10) is necessary. Either the constraining value of 1 could be changed to, for example, .999 or an approximation to the distribution could be used that truncates the limits. In either case, the loss of accuracy should be insignificant. 


$$
\begin{array}{cc}
\partial g_{t} / \partial A=-\partial \bar{X}_{t} / \partial A-N_{t}(1) \partial \sigma_{X_{t}} / \partial A<0, & t=1, \cdots, T \\
\partial g_{t+j} / \partial E_{t}=-\partial \bar{X}_{t+j} / \partial E_{t}-N_{t+j}(1) \partial \sigma_{X_{t+j}} / \partial E_{t} & \\
=-\partial f_{t+j} / \partial E_{t}=-e^{j} c & j=0, \cdots, T-t \\
\partial g_{t+j} / \partial L_{t}=-\partial \bar{X}_{t+j} / \partial L_{t}-N_{t+j}(1) \partial \sigma_{X_{t+j}} / \partial L_{t} & t=1, \cdots, T \\
=-\partial f_{t+j} / \partial L_{t}=e^{j} & \\
\partial g_{t} / \partial S=1, \quad t=1, \cdots, T &
\end{array}
$$

The sign of the term $\partial f_{t} / \partial A$ is ambiguous because $N_{t}\left(\rho_{t}\right)$ is negative.

A maximum for (13) is characterized by (14) through (29) if the objective function and constraints are concave in the decision variables. Provided $C(S)$ exhibits constant or increasing costs, the objective function is obviously concave. For constraint $f_{t}$,

$$
\partial^{2} f_{t} / \partial A^{2}=N_{t}\left(\rho_{t}\right) \partial^{2} \sigma_{X_{t}} / \partial A^{2}=\left(N_{t}\left(\rho_{t}\right) / \sigma_{X_{t}}\right) \sum_{j=0}^{t-1} e^{2 j} \sigma_{s_{t-j}}^{2} b^{2}<0
$$

and all other second partials are zero so $f_{t}$ is concave. Similarly, for $g_{t}, \partial^{2} g_{t} / \partial A^{2}=-N(1) \partial^{2} \sigma_{X_{t}} / \partial A^{2}<0$ and all other second partials are zero so $g_{t}$ is concave.

\section{Appendix B}

Geometric Brownian motion was introduced by Samuelson [20] to describe the movement in stock prices. The motion modifies the more familiar arithmetic Brownian motion introduced by Bachelier [3] and then by Einstein [8], by avoiding the situation where the random variables have a significant probability of being negative. Solar radiation, like stock prices, does not take on negative values.

Brownian motion (arithmetic or geometric) implies that if the random variable $s_{t}$ is known with certainty at time $t$, the random variable $s_{t+1}$ is known in the probability sense as it relates to $s_{t}$. Arithmetic Brownian motion implies that the probability of $s_{t+1}$, increasing or decreasing by one unit from $s_{t}$ is equal and independent of $s_{t}$. Geometric Brownian motion implies that the probability of $s_{t+1}$, increasing or decreasing one percentage unit from $s_{t}$, is equal and independent of $s_{t}$.

Given that $s_{t}$ and $s_{t+1}$ are random variables (such as the level of incoming solar radiation on successive days) that can be described by a geometric Brownian distribution, then the sequence of ratios $\left(z_{1}, z_{2}, \ldots\right)$ where $z_{t+1}=s_{t+1} / s_{t}$ for $(t=0,1,2, \ldots)$ are independent and identically log-normally distributed random variables described by:

$$
z_{t+1} \sim \Lambda\left(\mu_{z}, \sigma_{z}^{2}\right) .
$$

Following Samuelson and Merton [21], $s_{t}$ can be replaced by a product of terms:

$$
s_{t}=s_{0} \cdot z_{1} \cdot z_{2} \cdots \cdot z_{t} \text {. }
$$

This in turn is equivalent to:

$$
s_{t}=s_{0}\left(s_{t} / s_{0}\right) \text {. }
$$

Chen $[7,1045]$ has shown that $s_{t} / s_{0}$ is log-normally distributed with the following mean and variance:

$$
s_{t} / s_{0} \sim \Lambda\left(t \mu_{z}, t \sigma_{z}^{2}\right)
$$

where $t$ is the number of periods between the initial observation $s_{0}$ and the predicted observation $s_{t}$. Therefore, from (43), the random variable, $\ln \left(s_{t} / s_{0}\right)=\left(\ln s_{t}-\ln s_{0}\right)$, is normally distributed with mean $t \mu_{z}$ and variance $t \sigma_{z}{ }^{2}$. Subsequently $\ln s_{t}$ is normally distributed with mean $t \mu_{z}+\ln s_{0}$ and variance $t \sigma_{z}{ }^{2}$. The distribution of any $s_{t}$ following $t$ periods after an initial period observation $s_{0}$ is:

$$
s_{t} \sim \Lambda\left(t \mu_{z}+\ln s_{0}, t \sigma_{z}^{2}\right) .
$$

The conditional density function of $s_{t}$ given $s_{0}$ is: 


$$
f\left(s_{t} / s_{0}\right)=\left[1 / s_{t} \sigma_{z}(2 \pi t)^{1 / 2}\right] \exp \left(-1 / 2 \sigma_{z}^{2} t\right)\left[\ln s_{t}-\left(t \mu_{z}+\ln s_{0}\right)\right]^{2} .
$$

Thus, the distribution of any $s_{t}$, given an initial value of $s_{0}$, is dependent on the parameters $s_{0}, t, \mu_{z}, \sigma_{z}^{2}$.

In order to obtain a useful estimate of the mean and variance of $s_{t}$, the moment generating function technique can be used. The moment generating function of $s_{t}$ is:

$$
\mathbf{M}_{s_{t}}(j)=\int_{0}^{\infty} \mathrm{e}^{s_{t}} f\left(s_{t} / s_{0}\right) \mathrm{d} s_{t} .
$$

The $k$ th order moment about the origin described by $\lambda_{k}{ }^{\prime}$ is:

$$
\lambda_{k}{ }^{\prime}=\mathrm{d}^{k} \mathrm{M}_{s_{t}}(j) / \mathrm{d} j^{k}=\mathrm{E}\left(s_{t}^{k} / s_{0}\right)=\int_{0}^{\infty} s_{t}^{k} f\left(s_{t} / s_{0}\right) \mathrm{d} s_{t} .
$$

Defining $v_{t}=\ln s_{t}$ yields $\ln s_{t}^{k}=k \ln s_{t}$ and $\mathrm{d} s_{t} / s_{t}=\mathrm{d} \ln s_{t}=\mathrm{d} v_{t}$. Substitution of $v_{t}$ into (44) produces the moment generating function of a normally distributed variable $v_{t}$, i.e.,

$$
\lambda_{k}{ }^{\prime}=\int_{-\infty}^{\infty} \mathrm{e}^{k v_{t}} \mathrm{~d} N\left(v_{t}\right)=\exp \left[k \mu_{v_{t}}+1 / 2 k^{2} \sigma_{v_{t}}^{2}\right]
$$

Since $\mu_{v_{t}}$ and $\sigma_{v_{t}}{ }^{2}$ are the mean and variance of $\ln s_{t}$, the $k$ th order moment about the origin is

$$
\lambda_{k}{ }^{\prime}=\exp \left[k t \mu_{z}+k \ln s_{0}+1 / 2 k^{2} t \sigma_{z}^{2}\right] .
$$

Therefore, the mean and variance of $s_{t}$ given $s_{0}, t, \mu_{z}$ and $\sigma_{z}^{2}$ are respectively:

$$
\begin{aligned}
& \mu s_{t}=\exp \left[t \mu_{z}+\ln s_{0}+1 / 2 t \sigma_{z}^{2}\right] \\
& \sigma_{s_{t}}{ }^{2}=\exp \left[2 t \mu_{z}+2 \ln s_{0}+2 t \sigma_{z}{ }^{2}\right]-\mu_{s_{t}}{ }^{2} .
\end{aligned}
$$

The estimates of $s_{0}, \mu_{z}$ and $\sigma_{z}{ }^{2}$ should be taken from a fairly large sample (years) for the same period each year. For example, $s_{0}$ might be estimated for the first day in each month using fifty years of data. The mean, $\mu_{z}$ (and variance $\sigma_{z}^{2}$ ) should be estimated by forming a sample beginning with $s_{1} / s_{0}$ of one month and ending with $s_{0}{ }^{\prime} / s_{t}$, where $s_{0}{ }^{\prime}$ is the initial period of the following month and $s_{t}$ is the last observation before $s_{0}{ }^{\prime}$. This method has the value of maintaining a fairly continuous trend throughout the year. If the number of periods for which $s_{0}$ is estimated is increased, the estimates of $\mu_{z}$ and $\sigma_{z}{ }^{2}$ become better at the expense of additional computing costs. If trends in any $s_{0}$ seem to exist, due to long term climatic changes, the choice of the estimate of $s_{0}$ becomes more complex.

\section{References}

1. Anderson, Dennis, "Models for Determining Least-Cost Investments in Electrical Supply." Bell Journal of Economics and Management Science, Spring 1972, 267-99.

2. Asbury, Joseph G. and Ronald O. Mueller, "Solar Energy and Electric Utilities: Should They be Interfaced?" Science, February $1977,445-50$.

3. Bachelier, L. "Theory of Speculation," (translation of 1900 French edition), in The Random Character of Stock Market Prices," edited by P. H. Cootner. Cambridge: The M.I.T. Press, 1967, pp. 17-28.

4. Ben-David, Shaul, et al., "Near Term Prospects for Solar Energy: An Economic Analysis." Natural Resources Journal, April 1977, 169-208.

5. Caputo, Richard S. "Solar Power Plants: Dark Horse in the Energy Stable," in Toward A Solar Civilization, edited by R. H. Williams. Cambridge: M.I.T. Press, 1978, pp. 73-94.

6. Charnes, A., and W. W. Cooper, "Deterministic Equivalents for Optimizing and Satisficing under Chance Constraints." Operations Research, January-February 1963, 18-39.

7. Chen, Andrew, H. Y., "A Model of Warrant Pricing in a Dynamic Market." Journal of Finance December $1970,1041-60$.

8. Einstein, Albert, "Über die von der molekularkinetischen Theorie der Wärme geforderte Bewegung von in ruhenden Flüssigkeiten suspendierten Teilchen." Annalen der Physik, 1905, 549-60.

9. Feldman, Stephen L. and Bruce Anderson, "Public Utility and Solar Energy Interface: An Assessment of Policy Options.” Energy Research and Development Administration, DSE/2523-1, Dec. 1976. 
10. Georgescu-Roegen, Nicholas, "Energy Analysis and Economic Valuation." Southern Economic Journal, April 1979, 1023-58.

11. Hamlen, Susan S. and William Hamlen, “A Distributed Lag Model to Predict Incoming Solar Radiation." Solar Energy, 1977, 217-18.

12. Hamlen, Susan S., William Hamlen, and John T. Tschirhart. "An Application of Chance Constrained Programming to the Problem of Efficient Solar Heating via Storage." Unpublished manuscript, University of Wyoming, August 1978.

13. Hamlen, Susan S., William Hamlen, and John T. Tschirhart, "Geometric Brownian Distribution of Solar Radiation with an Economic Application." Solar Energy, 1978, 469-75.

14. Kreider, Jan and Frank Kreith. Solar Heating and Cooling. New York: McGraw-Hill, 1975.

15. Lorsch, Harold G., "Implications of Residential Solar Heating on Electric Utilities." Franklin Institute Research Laboratories Report No. F-C4209, Philadelphia, PA, May 1976.

16. Marchand, M. G., "Pricing Power Supplied on an Interruptible Basis." European Economic Review, October 1974, 263-74.

17. Meyer, Robert A., "Monopoly Pricing and Capacity Choice Under Uncertainty." American Economic Review, June 1975, 326-37.

18. Panzar, John C. and David S. Sibley, "Public Utility Pricing Under Risk: The Case of Self-Rationing." American Economic Review, December 1978, 888-95.

19. Roach, Fred, Scott Noll, and Shaul Ben-David, "Passive and Active Residential Solar Heating: A Comparative Economic Analysis of Select Designs." Los Alomos Scientific Laboratory Reprint LA-UR-79-57, 1979.

20. Samuelson, Paul, "Rational Theory of Warrant Pricing," Industrial Management Review, Spring 1965 (also in The Random Character of Stock Market Prices, edited by P. H. Cootner, 507-25, Cambridge: the M.I.T. Press, 1967).

21. Samuelson, Paul and Robert Merton, "A Complete Model of Warrant Pricing that Maximizes Utility." Industrial Management Review, Winter 1969, 17-46.

22. Schiflett, Mary and John V. Zuckerman, "Solar Heating and Cooling: Stats and Municipal Legal Impediments and Incentives." Natural Resources Journal, April 1978, 313-36.

23. Steiner, Peter O., "Peak Loads and Efficient Pricing." Quarterly Journal of Economics, November 1957, $585-610$

24. Telson, Michael L., "The Economics of Alternative Levels of Reliability for Electric Power Generation Systems." Bell Journal of Economics, Autumn 1975, 670-94.

25. Tschirhart, John T., and Frank Jen, "Behavior of a Monopolist Offering Interruptible Service." Bell Journal of Economics, Spring 1979, 244-58.

26. Tybout, R. A., and G. O. G. Löf, "Solar House Heating." Natural Resources Journal, April 1970, $268-326$ (an abbreviated version may be found in Solar Energy, 1973, 253-278).

27. U.S. Senate Committee on Energy and Natural Resources, The National Energy Act, January 1979, Publication No. 96-1, U.S. Government Printing Office, Washington, D.C.

28. Wenders, John T. and Lester D. Taylor, "Experiments in Seasonal-Time-of-Day Pricing of Electricity to Residential Users.” Bell Journal of Economics, Autumn 1976, 531-52. 